\title{
Migration und Entwicklung : Zögern der Schweizer Politik
}

\section{Gérard Perroulaz}

\section{(2) OpenEdition \\ 12 Journals}

Electronic version

URL: http://journals.openedition.org/sjep/359

DOI: 10.4000/sjep.359

ISSN: 1663-9677

Publisher

Institut de hautes études internationales et du développement

Printed version

Date of publication: 1 décembre 2008

Number of pages: $257-274$

ISBN: 978-2-940415-08-3

ISSN: 1660-5926

\section{Electronic reference}

Gérard Perroulaz, « Migration und Entwicklung : Zögern der Schweizer Politik», Schweizerisches Jahrbuch für Entwicklungspolitik [Online], 27-2 | 2008, Online erschienen am: 31 Mai 2010, abgerufen am 08 September 2020. URL : http://journals.openedition.org/sjep/359 ; DOI : https://doi.org/ 10.4000/sjep.359 


\title{
Migration und Entwicklung: Zögern der Schweizer Politik
}

\author{
Gérard Perroulaz*
}

\section{Einführung}

Im Rahmen der aktuellen Debatten über die Beziehungen zwischen Migration und Entwicklung weisen mehrere vor kurzem erschienene Studien nach, dass die Migrationspolitik der Länder des Nordens Folgen für die Entwicklungsländer hat ${ }^{1}$. Diese Studien unterstreichen die positiven Beiträge der Migration für die Entwicklungsländer, aus denen viele Migranten stammen, und verweisen nicht nur auf die negativen Aspekte (Abwanderung qualifizierter Arbeitskräfte, durch Verbrecherbanden organisierte illegale Migration, Menschenhandel). Hingegen hat sich die politische Migrationsdebatte lange Zeit auf Asylfragen und auf die Integration der Migranten in den Ländern des Nordens fokussiert.

Mehrere Studien jüngeren Datums, namentlich die Arbeiten des Entwicklungszentrums der Organisation für Entwicklung und Zusammenarbeit $(\mathrm{OECD})^{2}$, verweisen auf die Bedeutung eines höheren Grades an Kohärenz zugunsten der Entwicklung. Dabei geht es nicht nur darum, zu vermeiden, dass eine Politik in einem Einsatzbereich der Entwicklung schade, sondern darum, bessere Synergien zwischen den verschiedenen Politiken zu erreichen, um deren positiven Effekte auf die Herkunftsländer der Migranten zu verstärken. Jedoch ist es zumindest aus zwei Gründen nicht möglich, zwischen der Politik der Entwicklungszusammenarbeit, der Wirtschaftspolitik (Beschäftigung, Handel, Investionen), der Migrationspolitik und der nationalen Politik zur Integration von Ausländern vollkommene Kohärenz zu erreichen. Zum einen sind die Ziele der diversen Politiken unterschiedlich. Migrationspolitik ist auf die Kontrolle der Migrationsströme, die Integration ausländischer Gebietsansässiger, die Asylpolitik und die Rückführung abgewiesener Asylbewerber ausgerichtet. Sie hat nicht Armutsbekämpfung zum Ziel, welche das erklärte Ziel der Entwicklungshilfeagenturen ist. Zum anderen betreffen die Hilfeflüsse nicht dieselben Länder wie die Migrationsströme ${ }^{3}$. Die Entwicklungshilfe geht eher an die ärmsten Länder, wohingegen die Direktinvestitionen im Ausland und die Handelsströme

* Forschungsbeauftragter am Institut de hautes études internationales et du développement (IHEID), Genf.

1 Zum Beispiel die laufenden Forschungsarbeiten am Entwicklungszentrum der OECD. Siehe auch die Arbeiten des Weltforums für Migration und Entwicklung (Global Forum on Migration and Development, GFMD).

2 Jeff Dayton-Johnson und Louka T. Katseli, Migration, aide et commerce: plus de cohérence en faveur du développement, Cahier de politique économique, Nr. 28, Paris, OCDE, 2006.

3 Siehe Denis Cogneau und Sylvie Lambert, L'aide au développement et les autres flux Nord-Sud: complémentarité ou substitution?, Document de travail, Nr. 251, Paris, Centre de développement de l'OCDE, 2006. Vgl. auch Kapitel 9 der OECD-Publikation La cohérence des politiques au service du développement: migrations et pays en développement, Paris, OCDE, 2007, 164 S. 
die Tendenz haben, sich auf eine Minderheit von Ländern mit mittlerem Einkommen zu konzentrieren. Die meisten Migrantinnen und Migranten, die in die OECD-Staaten anreisen, kommen nicht aus den ärmsten Ländern. Daraus lässt sich schliessen, dass die ärmsten Länder, die am meisten von der öffentlichen Entwicklungshilfe abhängen, relativ wenig Nutzen aus den positiven Effekten der Migration - wie die Geldüberweisungen und die Erlangung von Kompetenzen anlässlich der Rückkehr der Migranten - ziehen.

In diesem Artikel stellen wir eingangs kurz die von der Migration betroffenen Akteure in der Schweiz vor ${ }^{4}$ und befassen uns dann ausführlicher mit der Rolle der Direktion für Entwicklung und Zusammenarbeit (DEZA). Der zweite Teil des Artikels ist auf die Analyse des Diskurses über den Zusammenhang zwischen Migration und Entwicklungszusammenarbeit fokussiert. Dabei werden die von der Schweiz geförderten internationalen Initiativen präsentiert und die Strategietexte der Entwicklungszusammenarbeit untersucht. Im dritten Teil legen wir die Schwierigkeiten der Schweizer Entwicklungszusammenarbeit dar, von den schriftlichen Absichtserklärungen zu operationellen Projekten im Migrationsbereich überzugehen. Danach analysieren wir die Spannungsfelder zwischen der Migrationspolitik und den anderen Politiken der Schweiz, die eine Auswirkung auf die Entwicklung haben. Schliesslich untersuchen wir anhand einiger auf internationaler Ebene veröffentlichter Studien, welche aktivere Rolle die Entwicklungszusammenarbeit spielen könnte, um die negativen Auswirkungen der Migration zu verringern, bzw. die positiven Effekte auf die Entwicklung der Herkunftsländer zu steigern.

\section{Überblick über die betroffenen Akteure in der Schweiz}

Unter den von der Migration in der Schweiz betroffenen Akteuren ist auf eidgenössischer Ebene zuerst das Bundesamt für Migration (BFM) zu nennen, das mit der Asylpolitik beauftragt und für Migrationsfragen zuständig ist. Das BFM unterhält in Verbindung mit den kantonalen Instanzen eine breite Palette von Aktivitäten im Migrationsbereich betreffend die Aufenthalts- und Arbeitsbewilligungen, die Integrationspolitik, die Einbürgerung, das Asylwesen und die Rückkehrhilfe. Im Bereich der internationalen Angelegenheiten hat das BFM bei der Initiative von Bern und beim Abschluss von Rückübernahmeabkommen mit den Herkunftsländern der Migranten eine Rolle gespielt.

Neben dem Bundesamt für Migration hat sich das Staatssekretariat für Wirtschaft (SECO) in den letzten Jahren für die Fragen der Geldüberweisungen von Migranten interessiert. Die Politische Abteilung IV des Eidgenössischen Departements für auswärtige Angelegenheiten (EDA) beschäftigt sich ihrerseits vor allem mit dem Folgeprozess des internationalen Dialogs im Migrationsbereich und mit dem Kampf gegen den Menschenhandel. Die Direktion für Entwicklung und Zusammenarbeit (DEZA) führte bis zum Jahr 2008 auch das Migrationsthema unter den zehn Schwerpunktthemen der Schweizer Entwicklungs-

4 Andere Beiträge im vorliegenden Dossier beschreiben die Tätigkeit einiger staatlicher Organe. Siehe insbesondere den Beitrag von Pietro Mona (Politische Abteilung IV Menschliche Sicherheit, Eidgenössisches Departement für auswärtige Angelegenheiten) sowie den Artikel von Eric Kaser und Saskia Schenker (Bundesamt für Migration, Eidgenössisches Justiz- und Polizeidepartement). 
zusammenarbeit auf. Wie wir sehen werden, betreffen die Projekte der DEZA aber vor allem die humanitäre Hilfe.

Schliesslich ist auch die Rolle der Eidgenössischen Kommission gegen Rassismus $(\mathrm{EKR})^{5}$ und der Eidgenössischen Kommission für Migrationsfragen $(\mathrm{EKM})^{6}$ hervorzuheben. Die letztgenannte ausserparlamentarische Kommission für MIgrationsfragen wurde vom Bundesrat am 1. Januar 2008 eingesetzt; sie ging aus der Zusammenlegung der früheren Eidgenössischen Ausländerkommission (EKA) und der Eidgenössischen Kommission für Flüchtlingsfragen (EKF) hervor.

Die Schweiz hat sich am internationalen Migrationsdialog beteiligt und unterstützt die Arbeit mehrerer internationaler Organisationen in diesem Bereich, und zwar das UN-Hochkommissariat für Flüchtlingsfragen (UNHCR), das Hochkommissariat für Menschenrechte (HCHR), die Internationale Arbeitsorganisation (IAO) betreffend die Arbeitsrechte und -bedingungen der Migranten sowie das Hilfswerk der Vereinten Nationen für die Palästinaflüchtlinge im Nahen Osten (UNWRA). Die Eidgenossenschaft unterhält besonders enge Beziehungen zur Internationalen Organisation für Migration (IOM) mit Sitz in Genf, deren Büro in Bern sie finanziert und mit der sie aktiv zusammenarbeitet. Diese Instanz ist besonders mit der Umsetzung der Rückkehrhilfeprogramme und der Errichtung von Migrationspartnerschaften beauftragt.

Unter den übrigen Akteuren spielen die Kantone namentlich bei der Erteilung von Arbeitsbewilligungen und der Regularisierung von Papierlosen eine bedeutende Rolle. Die in erster Linie betroffenen Akteure, das heisst die Migranten selber, sind über ihre Verbände ebenfalls Gesprächspartner. Die Nichtregierungsorganisationen (NRO) führen humanitäre Einsätze bei Katastrophen und Konflikten durch. Sie treten aber auch für die Menschenrechte und das Asylrecht ein, indem sie gegen die Verschärfung der Aufnahmeverfahren der Asylbewerber in der Schweiz vorgehen und den Asylsuchenden konkrete Unterstützung und individuelle Beratung bei der Rückkehrhilfe zukommen lassen. Zu den wichtigsten im Migrationsbereich tätigen Nichtregierungsorganisationen gehören das Schweizerische Rote Kreuz, Caritas, das Hilfswerk der Evangelischen Kirchen der Schweiz (HEKS), die Stiftung Terre des Hommes und das Centre protestant social. Einige NRO - wie die Dachorganisation Schweizerische Flüchtlingshilfe, Solidarités sans Frontières oder die Internetplattform $<$ http://www.humanrights.ch $>$ - sind auf dem Gebiet der Anwaltschaft zugunsten des Asylrechts oder der Ausländer spezialisiert. Viele andere Organisationen, wie beispielsweise das Centre de contact Suisses-Immigrés, setzen sich im Integrationsbereich ein.

\section{Engere Verbindung zwischen Migration und Entwicklungszusammenarbeit und Beteiligung der Schweiz am internationalen Dialog}

Bereits Ende der 80er Jahre fingen einige Initiatitiven vorsichtig an, internationale Entwicklungszusammenarbeit und Migration miteinander $\mathrm{zu}$ verbinden. Zuvor waren die Einwanderung ausländischer Arbeitnehmer in der Schweiz, die

\footnotetext{
$<$ http://www.ekr-cfr.ch $>$.

$<$ http://www.ekm.admin.ch>.
} 
Asylverfahren und die humanitäre Hilfe für Flüchtlinge in Krisengebieten getrennt verwaltet worden. In der Schweiz lässt sich der Zeitpunkt, zu dem erstmals eine Verbindung zwischen Entwicklungszusammenarbeit und Migration hergestellt wurde, genau festlegen: Und zwar ersuchte ein Postulat von Nationalrat Ernst Mühlemann ${ }^{7}$ vom 6. Dezember 1988 den Bundesrat, der Entwicklungszusammenarbeit mit der Türkei erhöhte Priorität einzuräumen. Nach einem plötzlichen Anstrom türkischer Asylbewerber mussten nämlich Mittel und Wege gefunden werden, um den grössten Teil dieser Asylanten zurückzuschaffen ${ }^{8}$. Der Krieg in den Staaten Ex-Jugoslawiens führte später dazu, die Beziehung zwischen humanitärer Hilfe und Ayslpolitik zu verstärken: Die humanitäre Hilfe sollte immer mehr dazu beitragen, die Bevölkerung in ihrem eigenen Land (durch Hilfe für die intern Vertriebenen) oder in den umgebenden Ländern zu unterstützen, um dadurch den Zustrom von Flüchtlingen nach Westeuropa aufzuhalten. Auch das wirtschaftliche Argument wird in diesem Zusammenhang herangezogen: Die mit der Präsenz von Asylbewerbern in der Schweiz verbundenen jährlichen Kosten seien viel höher als die Ausgaben für die Aufnahme von Flüchtlingen in Lagern in den an die Krisengebiete angrenzenden Ländern oder Regionen.

In den 90er Jahren machte sich das Bedürfnis nach einer besseren Koordination zwischen den für Migrationsfragen zuständigen Bundesämtern bemerkbar. 1992 setzte der Bundesrat die Interdepartementale Arbeitsgruppe für Migrationsprobleme ein, die 1998 zur Interdepartementalen Arbeitsgruppe Migration (IAM) umbenannt wurde, und in der alle eidgenössischen Departemente vertreten sind. 1995 wurde die Interdepartementale Leitungsgruppe Rückkehr (ILR) geschaffen. Sie wird gemeinsam vom Bundesamt für Migration und von der DEZA geleitet und umfasst Vertreter der Politischen Abteilung IV (EDA) sowie der Internationalen Organisation für Migration (IOM).

\section{Eine im Migrationsbereich unerlässliche internationale Zusammenarbeit}

Auch wenn die Migrationspolitik ein wichtiger Bestandteil der nationalen Souveränität eines jeden Staates ist, erscheint eine verbesserte internationale Abstimmung unerlässlich, um die Migrationsströme $\mathrm{zu}$ bewältigen und $\mathrm{zu}$ steuern ${ }^{9}$. Die Schweiz hat diese internationale Abstimmung mit der Lancierung der Berner Initiative im Jahr 2001 durch das Bundesamt für Flüchtlinge (heute Bundesamt für Migration) weitgehend gefördert. In diesem Rahmen ermöglichten zwei internationale Konferenzen in Bern in den Jahren 2003 und 2004, einen Dialog zwischen Herkunfts-, Transit- und Zielländern der Migranten einzuleiten ${ }^{10}$, und trugen zur Fertigstellung der Internationalen Agenda für Migrationsmanagement bei. Diese Agenda setzt die Leitlinien der internationalen Zusammenarbeit im Migrationsbereich fest und anerkennt die Bedeutung

7 Nationalrat (FDP, Thurgau) von 1983 bis 1999, Mitglied der Aussenpolitischen Kommission von 1995 bis 1999.

8 DEZA, „Internationale Zusammenarbeit und Migration“, Entwicklungspolitische Schriften, Nr. 4, Bern, DEZA, April 2001.

9 Siehe insbesondere die DEZA-Schrift „Internationale Zusammenarbeit und Migration“, op. cit., oder das DEZA-Magasin Eine Welt, Nr. 2, Mai 1999.

10 Zur Initiative von Bern siehe die Informationen auf der Website des Bundesamtes für Migration unter : <http://www.bfm.admin.ch/bfm/de/home/themen/internationales.html> > Multilaterales. 
einer verstärkten Zusammenarbeit. Als nichtbindendes Instrument ist die Internationale Agenda für Migrationsmanagement das Ergebnis von Beratungen und nicht von Verhandlungen unter Staaten.

Parallel zu diesem von der Schweiz initiierten Prozess schlug der frühere UNGeneralsekretär Kofi Annan bereits im Jahr 2002 eine Reflexion über Migrationsfragen im Rahmen der Vereinten Nationen vor. Eine Arbeitsgruppe der UNO verwies in einem im Jahr 2003 veröffentlichten Bericht auf die institutionellen Lücken und die Unzulänglichkeit der internationalen Normen im Migrationsbereich (Doyle-Bericht) ${ }^{11}$. Der Bericht untersuchte insbesondere die mögliche Rolle der internationalen Organisationen und schlug einige Varianten für die Zukunft vor, darunter die Möglichkeit der Einsetzung einer internationalen Agentur, welche die Tätigkeiten der Vereinten Nationen im Migrationsbereich koordinieren sollte, da sich die bestehenden Organisationen (UNHCR, IAO und IOM, letztere mit einem etwas besonderen Statut, da sie nicht dem UN-System angehört) nur teilweise mit diesem Aspekt befassen ${ }^{12}$.

Die Weltkommission für Internationale Migration (Global Commission on International Migration, GCIM) wurde im Anschluss an den Doyle-Bericht im Dezember 2003 im Beisein von Bundesrätin Micheline Calmy-Rey geschaffen. Diese vom Schweizer Rolf K. Jenny geleitete Expertenkommission veröffentlichte am 5. Oktober 2005 ihren Schlussbericht ${ }^{13}$. Darin stellte die Kommission fest, dass die Migrationspolitik auf gemeinsamen Zielen und einer gemeinsamen Vision beruhen müsse, um die Vorteile der internationalen Migrationsbewegungen zu maximieren und die negativen Auswirkungen auf ein Minimum zu reduzieren. Ferner schlug die Kommission folgende sechs Handlungsprinzipien vor: Aus freier Wahl auswandern; die Wirkung auf die Wirtschaft und die Entwicklung verstärken; die Frage der irregulären Migration behandeln; den gesellschaftlichen Zusammenhalt durch die Integration fördern; die Rechte der Migranten schützen; die Migrationsgouvernanz stärken. Diese Prinzipien gingen mit Empfehlungen einher, die weiterhin völlig aktuell sind.

Die Reflexion wurde auf internationaler Ebene mit den Arbeiten des Hochrangigen UN-Dialogs über Migration und Entwicklung (UN-High Level Dialogue on Migration and Development) im September 2006 weitergeführt. Diese Konferenz bekräftigte die Notwendigkeit, den Dialog unter den Staaten fortzusetzen. Hierzu schlug der UN-Generalsekretär die Einsetzung des Weltforums für Migration und Entwicklung (Global Forum on Migration and Development, GFMD) vor, statt eine neue internationale Institution zu gründen oder eine der bestehenden Organisationen zur Koordinatorin der Tätigkeiten in diesem Bereich zu ernennen. Dieses informellere Forum beruht auf der freiwilligen Teilnahme der Staaten und der Einbeziehung der Zivilgesellschaft. Es soll keine ausgehandelten Ergebnisse oder verbindlichen Beschlüsse hervorbringen, sondern einen Rahmen für den Dialog sowie zum Austausch von Erfahrungen und

11 Michael Doyle, Report to the Secretary General on Migration, New York, 2003, 41 S.

12 Der Doyle-Bericht erwähnte andere mögliche Optionen, wie die Ernennung einer bestehenden Agentur zur Koordinierung der Aktionen im Migrationsbereich, die Integration der IOM ins UN-System als Sonderorganisation, die Annahme internationaler Abkommen zur Regulierung der Migrationssströme (wie die Handelsabkommen) oder die Einsetzung einer internationalen Kommission.

13 Commission mondiale sur les migrations internationales (CMMI), Les migrations dans un monde interconnecté : nouvelles perspectives d'action, 2005, <http://www.gcim.org>. 
guten Praktiken bieten. Das erste Treffen des Hochrangigen UN-Dialogs fand im Juli 2007 mit Vertretern aus 150 Staaten in Brüssel statt; ein weiteres Treffen folgte im Oktober 2008 in Manila ${ }^{14}$. Die Schweiz beteiligt sich aktiv an den Arbeiten des Forums durch die Politische Abteilung IV des EDA. Mit nationalen Schwerpunkten soll ein umfassenderer und kohärenterer Ansatz der Thematik ,Migration und Entwicklung“ erreicht werden.

\section{Entwicklungshilfe als Mittel zur Eindämmung der Migrationsströme?}

Die Schweiz hat zwar interessante Initiativen zur Förderung des Dialogs auf internationaler Ebene eingeleitet, jedoch hatte sie Mühe, einige Vorschläge in Aktionen bilateraler Entwicklungszusammenarbeit umzusetzen. Die DEZA nahm in diesem Bereich eine relativ defensive Haltung ein, indem sie anführte, dass die öffentliche Entwicklungshilfe die Migrationsströme nicht aufzuhalten vermöge. Auch widersetzte sie sich der Konditionalität der Hilfe, mit dem Argument, dass man beispielsweise die Hilfe für Länder, die bei der Rückführung von eigenen Staatsangehörigen nicht kooperationsbereit seien, nicht abbrechen dürfe. Ferner betonte sie, die Entwicklungszusammenarbeit dürfe nicht für Ziele wie die massive Rückschaffung von Ausländern instrumentalisiert werden. Aufgrund dieser Haltung war die DEZA nicht imstande, genügend Projekte im Bestreben zu fördern, gewisse positive Aspekte der Migration zu maximieren, wohingegen in zahlreichen Forschungsarbeiten und Studien interessante Lösungsansätze angesprochen werden. In mehreren um das Jahr 2000 herum veröffentlichten Dokumenten führte die DEZA die Entwicklungszusammenarbeit als wirksames Mittel zur Bekämpfung bestimmter Migrationsursachen $a^{15}$. Parallel dazu wies die DEZA aber ständig darauf hin, dass auch wenn sie ihre gesamte Hilfe auf die Herkunftsländer der Migranten in der Schweiz konzentrieren würde, die Entwicklungszusammenarbeit nicht die Kapazität hätte, die Migrationsströme aufzuhalten ${ }^{16}$.

In seiner Botschaft über die Weiterführung der technischen Zusammenarbeit und der Finanzhilfe zu Gunsten von Entwicklungsländern vom Jahr 2003 macht der Bundesrat folgende Ausführungen zum Thema Migration: „Der Hauptbeitrag der Entwicklungszusammenarbeit an die Problemlösung im Bereich der Migration ist darin zu sehen, dass sie längerfristig die Ursachen der notgedrungenen und erzwungenen Migration - hauptsächlich die Armut - abbauen hilft und damit eine generalpräventive Wirkung bezüglich der Migration entfaltet. Verbesserter Zugang zu Arbeitsmöglichkeiten, zum Ausbildungs- und Gesundheitswesen, zu Trinkwasser und zur ungehinderten Teilhabe am politischen Geschehen wie auch die nachhaltige Bewirtschaftung der vorhandenen Ressourcen tragen mit dazu bei, dass Auswanderung für viele Menschen keine valable Option mehr dar-

14 Rapport de la première session du Forum sur la migration et le développement, Bruxelles, 9-11 juillet $2007,30 \mathrm{~S}$

15 Das vollständigste Dokument der DEZA zu Migrationsfragen ist die bereits erwähnte Schrift „Internationale Zusammenarbeit und Migration“ aus der Reihe Entwicklungspolitische Schriften, Nr. 4, 2001. Siehe auch das Dossier „Migration und Entwicklungszusammenarbeit“ im DEZA-Magasin Eine Welt, Nr. 2, 1999.

16 Die Studien der OECD weisen nach, dass die Korrelation zwischen Entwicklungshilfe und Wirtschaftswachstum schwach ist und dass das Wirtschaftswachstum im Süden - ausser auf sehr lange Sicht - keinen Rückgang der Migrationsströme nach sich zieht. 
stellt.“" Die Migration wird hier grundsätzlich als ein „Problem“ angesehen, während im Allgemeinen anerkannt wird, dass die Migration eine unumgängliche Realität darstellt und dass sie in den kommenden Jahrzehnten anhalten wird.

In derselben Botschaft räumt der Bundesrat indessen ein, dass die geringen Mittel der Schweiz für die Entwicklungszusammenarbeit nicht die Migrationsströme aufzuhalten vermögen, dass das Problem der unkontrollierten Migration durch einzelstaatliche Massnahmen der Länder des Nordens allein nicht zu bewältigen sei, und dass gemeinsame Anstrengungen der internationalen Staatengemeinschaft notwendig seien. Im Zusammenhang mit der Rückübernahme von eigenen Staatsangehörigen durch die Staaten hält der Bericht Folgendes fest: „Die Entwicklungszusammenarbeit mit den Problemen in diesem Bereich zu verknüpfen und diese gegenüber Staaten zu suspendieren oder abzubrechen, welche nicht genügend oder gar nicht kooperationsbereit sind, ist weder eine realistische, noch eine nutzenbringende Massnahme." 18 Damit widersetzt sich der Bundesrat einer Konditionalisierung der Hilfe. Nach Erachten des Bundesrates sei es auch nicht sinnvoll, für eine Konzentration der Hilfe auf die hauptsächlichen Herkunftsländer der Migranten einzutreten, umso mehr, als es sich bei diesen Ländern eher um Länder mit mittlerem Einkommen handle, wohingegen die Schweizer Entwicklungszusammenarbeit prioritär für die ärmsten Länder bestimmt sei.

\section{Botschaft über die Entwicklungszusammenarbeit 2008-2012: Migration im Hintergrund}

Trotz der Vielzahl der Studien auf internationaler Ebene, welche auf die Zusammenhänge zwischen Migration und Entwicklung hinweisen, wird das Migrationsphänomen in der neuen Botschaft des Bundesrats über die Weiterführung der technischen Zusammenarbeit und der Finanzhilfe zugunsten von Entwicklungsländern nur kurz mit folgenden Worten beschrieben: „Rund 200 Millionen Menschen leben ausserhalb ihrer Heimat. Das sind beinahe 3 Prozent der Weltbevölkerung. Tendenz steigend. Aus wirtschaftlichen Motiven oder wegen politischer und sozialer Konflikte verlassen viele Menschen ihr Heimatland in Richtung Industrie- und Schwellenländer. Die Süd-Süd-Migration ist allerdings noch grösser. Aufgrund der Migration sind bedeutende Immigranten-Gemeinschaften entstanden - ein Phänomen, dass sowohl Risiken (z.B. Eingliederungsprobleme, ,Braindrain') als auch Chancen birgt (z.B. Know-how-Transfer und Geldtransfer der Emigranten in ihre Heimatländer). Das Volumen der Geldüberweisungen aus diesen ,Diasporas ' in die Heimatländer übersteigt die gesamte öffentliche Entwicklungszusammenarbeit um ein Mehrfaches. Es ist eine Herausforderung, Anreize für eine gezielte Nutzung dieses Potenzials zu schaffen." ${ }^{19}$

Migration, die im Rahmenkredit von 2003 zu den zehn Schwerpunktthemen der DEZA gehörte, wird in der neuen Botschaft des Bundesrates nicht mehr unter den sieben Schwerpunktthemen der DEZA aufgeführt. Die Botschaft merkt jedoch an, dass die DEZA sich auch mit regionaler Zusammenarbeit, mit

17 Bundesrat, Botschaft über die Weiterführung der technischen Zusammenarbeit und der Finanzhilfe zu Gunsten von Entwicklungsländern, vom 28. Mai 2003 (BB1 2003 4625), Zitat S. 4658.

18 Ibid., S. 4660.

19 Bundesrat, Botschaft über die Weiterführung der technischen Zusammenarbeit und der Finanzhilfe zugunsten von Entwicklungsländern, vom 14. März 2008 (BB1 2008 2959), Zitat S. 2966-2967. 
Konfliktprävention und mit Migration in den zwölf Schwerpunktländern der Schweizer Entwicklungszusammenarbeit befassen kann. Im Juni 2008 äusserte Bundesrätin Micheline Calmy-Rey die Ansicht, globale Entwicklungen wie die Nahrungsmittelkrise, der Wassermangel, die Migrationsströme und Staaten mit Fehlschlägen seien Auslösungsfaktoren für die grosse Umorganisation der DEZA im Sommer des gleichen Jahres gewesen ${ }^{20}$. Im Eidgenössischen Departement für auswärtige Angelegenheiten (EDA) wurde ein Prozess zur Vorbereitung von drei Strategievorschlägen in den Schwerpunktbereichen Klimawandel, Ernährung und Migration eingeleitet. Auf der Jahreskonferenz der DEZA und des SECO im August 2008 präsentierte die Vorsteherin des EDA jedoch nur noch Aktionen in zwei Schwerpunktbereichen: Ernährungssicherheit und Klimawandel ${ }^{21}$. Wie wir nachstehend sehen werden, sind die operationellen Projekte im Migrationsbereich im Vergleich zu anderen Schwerpunktthemen seit jeher von eher bescheidener Tragweite gewesen.

\section{Schwieriger Übergang zur operationellen Umsetzung}

Auch wenn die Schweiz sehr aktiv an der internationalen Reflexion über die Zusammenhänge zwischen Migration und Entwicklung teilgenommen hat, konnte diese Thematik nur mit Schwierigkeit auf der Ebene der operationellen Abteilungen umgesetzt werden. Im Migrationsbereich haben vor allem die für die Hilfe an die südosteuropäischen Länder zuständigen Abteilungen Projekte - oft in Verbindung mit der Rückkehrhilfe - in folgenden Hauptaktionsfeldern entwickelt :

- Programme humanitärer Hilfe für Flüchtlinge und intern Vertriebene in verschiedenen Krisengebieten. In den Jahren 2006 und 2007 waren die hauptsächlichen Einsatzgebiete der DEZA: Bosnien-Herzegowina, der Nahe Osten (Hilfe für die palästinensischen Flüchtlinge), Armenien, Thailand (Hilfe für die birmanischen Flüchtlinge), Afghanistan (Rückkehrhilfe), der Irak (Hilfe für die intern Vertriebenen und Rückkehrhilfe), Kolumbien (Hilfe für die intern Vertriebenen), Algerien (Hilfe für die Sahraoui-Flüchtlinge) und der Tschad (Hilfe für die Flüchtlinge aus Darfur).

- Hilfe für Migranten in Transitländern. Nehmen wir das Beispiel Marokkos. Dieses Land dient immer mehr Migranten aus Afrika südlich der Sahara, die nach Europa zu gelangen versuchen, als Transitland. Die betroffenen Migranten auf der Durchreise in illegaler Situation geniessen keinen Rechtsschutz und sind nicht imstande, ihre Grundbedürfnisse an Nahrung und Unterkunft zu decken. Durch in Zusammenarbeit mit NRO wie Caritas durchgeführte Nothilfeprojekte können die Grundbedürfnisse der Migranten (Nahrung, medizinische Behandlung, Unterkunft und psychologische Betreuung) gedeckt werden. Médecins du Monde lässt den Migranten ärztliche Behandlung und einen Rechtsschutz zukommen. Die Internationale Organisation für Migration (IOM) bietet den Migranten Hilfe für die Rückkehr in ihr Herkunftsland an.

- Rückkehrhilfe. Die DEZA beteiligt sich an den Rückkehrhilfeprogrammen, vor allem an der Strukturhilfe vor Ort, mit der Finanzierung und der Zusammenarbeit des Bundesamtes für Migration.

20 EDA, Pressemitteilung, 9. Juni 2008.

21 DEZA, Jahreskonferenz der Entwicklungszusammenarbeit, Pressemitteilung, 22. August 2008. 
- Programme zur Prävention irregulärer Migration. Diese Programme umfassen insbesondere die Aufführung von Theaterstücken in Moldawien, Weissrussland und der Ukraine, um junge Frauen und ihre Familien für die Gefahren der irregularen Migration zu sensibilisieren.

- Hilfe vor Ort für die Opfer von Menschenhandel. Die lokalen Behörden sowie Nichtregierungsorganisationen erhalten finanzielle Unterstützung für die Wiedereingliederung von Opfern von Menschenhandel in ihrem Herkunftsland (medizinische Behandlung, psychologische Unterstützung, Ausbildung und Rückkehrhilfe). Diese Programme werden vor allem im Balkan, in Russland, Moldawien, in der Ukraine und in Weissrussland durchgeführt.

Wir verfügen über keine genauen Angaben bezüglich der Beträge öffentlicher Entwicklungshilfe, die zur Finanzierung von Projekten im Migrationsbereich bestimmt sind. Diesbezügliche Schätzungen sind zudem schwierig, da der Migrationsbereich nicht klar abgegrenzt ist. So wird zum Beispiel ein Programm, das in einer benachteiligten Region Arbeitsplätze schafft, eine indirekte positive Wirkung auf die Migration haben, indem es Menschen, die andernfalls versucht wären, auszuwandern, eine Aussicht auf Beschäftigung bietet. Jedoch lassen sich anhand der bruchstïckhaften Daten, die betreffend die Projekte der DEZA zur Verfügung stehen, drei Feststellungen zur Auswirkung dieser Projekte auf die Migration machen:

1. Die wichtigsten Programme für die Hilfe im Migrationsbereich betreffen die humanitäre Hilfe. Von der gesamten öffentlichen Entwicklungshilfe macht die humanitäre Hilfe rund ein Fünftel aus, und 80 Prozent der Hilfe sind für längerfristige Projekte der Entwicklungszusammenarbeit bestimmt. Gemäss der verfügbaren Information scheint dieses Verhältnis im Migrationsbereich umgekehrt zu sein. Die meisten Projekte der DEZA betreffen die Nothilfe (für intern Vertriebene und Flüchtlinge in den Nachbarländern), die Rückkehrhilfe für abgewiesene Asylbewerber und den Kampf gegen den Menschenhandel. Die DEZA führt in ihren Texten häufig das Argument an, es sei vorzuziehen, den Flüchtlingen und Vertriebenen vor Ort oder im Krisengebiet selbst zu helfen, anstatt das Risiko des Eintreffens von Flüchtlingsströmen in den Ländern des Nordens in Kauf nehmen zu müssen. Daneben wird auch das ökonomische Argument angeführt: Es sei weniger aufwändig, einem Flüchtling in einem Nachbarland des Konflikts zu Hilfe zu kommen, als die Bedürfnissen eines Asylbewerbers in der Schweiz zu decken.

2. Der Grossteil der in der öffentlichen Entwicklungshilfe integrierten Ausgaben hat nichts mit wirklicher Entwicklungshilfe im Migrationsbereich zu tun. 2007 belief sich die öffentliche Entwicklungshilfe der Schweiz auf 2020 Millionen Franken; davon wurden vom Bundesamt für Migration 9,3 Millionen Franken für Rückkehrhilfe und 182,9 Millionen Franken für Ausgaben in der Schweiz (Kosten im Zusammenhang mit der Präsenz von Asylbewerbern in der Schweiz im ersten Aufenthaltsjahr) aufgebracht ${ }^{22}$. Auch wenn die Rückkehrhilfe im Fall der Strukturhilfe vor Ort als Entwicklungshilfe angesehen werden kann, so

22 Zahlen der DEZA, siehe <http://www.deza.admin.ch/de/Home/Die_DEZA/Zahlen_und_Fakten>.

Zum Vergleich sei angeführt, dass die Überweisungen der ausländischen Arbeitnehmer in der Schweiz in ihre Heimatländer im Jahr 2005 auf 9 Milliarden Dollar (einschliesslich der Überweisungen in Nachbarländer und von Grenzgängern) veranschlagt wurden. 
haben die mit der Präsenz des Asylbewerbers in der Schweiz verbundenen Ausgaben jedoch keine effektive Auswirkung auf die Entwicklung seines Herkunftslandes.

3. Bei den Projekten der DEZA im Bereich der längerfristigen Entwicklungszusammenarbeit handelt es sich in den meisten Fällen um Pilotprojekte mit einem sehr geringen Betrag ${ }^{23}$.

Somit ist anzumerken, dass die Entwicklungshilfe noch allzu oft als ein Mittel zur Eindämmung der Migration und zur Bekämpfung des Menschenhandels betrachtet wird. Die Migration als solche wird vor allem als ein Problem angesehen, gegen das man angehen muss. Es gibt zu wenig operationelle Projekte, um die positiven Wirkungen der Migration zu stärken und den Beitrag der Migranten sowohl in ihrem Herkunftsland als auch im Aufnahmeland zu fördern. Wir geben am Schluss dieses Artikels einige Hinweise für mögliche Aktionen im Zusammenhang mit der längerfristigen Entwicklungszusammenarbeit an.

\section{Spannungen zwischen der Migrationspolitik und anderen Schweizer Politiken}

Es ist unvermeidlich, dass sich zwischen den verschiedenen von Migrationsfragen betroffenen Departementen, und sogar im Innern der einzelnen Ämter Spannungsfelder ergeben. Nachstehend stellen wir einige der Widersprüche zwischen Asylpolitik, Migrationspolitik, humanitärer Politik und Politik zum Schutz der Menschenrechte heraus.

\section{Eine zweistufige Zulassungspolitik}

Seit der Einführung der Politik der „Drei Kreise“ im Jahr 1991 und bei allen späteren Anpassungen der betreffenden Gesetze hat die Migrationspolitik qualifizierte Migranten aus europäischen Ländern bevorzugt. Seit Inkrafttreten des bilateralen Abkommens über den freien Personenverkehr mit der Europäischen Union am 1. Juni 2002 werden Personen aller Qualifikationen aus EU- und EFTA-Staaten in der Schweiz prioritär aufgenommen, sofern sie dort eine Beschäftigung finden. Der Zugang zum Schweizer Arbeitsmarkt wird für qualifizierte Personen aus Drittstaaten schwierig, und wenig qualifizierten Personen aus aussereuropäischen Ländern bleibt die Tür versperrt. Staatsangehörigen aus armen Ländern, die Arbeit suchen und ihre Familie im Herkunftsland unterstützen wollen, bleibt somit keine andere Wahl, als zu versuchen, auf illegalem Wege in die Schweiz zu gelangen. Die Folge dieser Schliessung ist die Entwicklung einer Schattenwirtschaft, die laut Schätzungen rund 100000 Arbeitnehmer ohne Rechtsstatus beschäftigt ${ }^{24}$. Ihre Anwesenheit entspricht einem realen

23 Hierzu sei als Beispiel das Projekt Re-connect in Höhe von 150000 Franken erwähnt, das von 2005 bis 2007 in Bosnien durchgeführt wurde. Das Projekt richtete sich an junge Bosniaker, die in Bosnien-Herzegowina oder im Ausland lebten und ihre Studien gerade beendet hatten. Die Teilnehmer konnten Lehrgänge in Bosnien absolvieren, wodurch sie eine gewisse Berufserfahrung erlangten. So konnte der Auswanderung vorgebeugt werden, und Jugendliche der Diaspora in der Schweiz konnten dank einer besseren Kenntnis der Verhältnisse vor Ort eine Rückkehr in ihr Land ins Auge fassen.

24 Siehe Claude Longchamp et al., Sans Papiers in der Schweiz: Arbeitsmarkt, nicht Asylpolitik ist entscheidend. Schlussbericht im Auftrag des Bundesamtes für Migration, Bern, GFS, Februar 2005. 
Bedarf der Schweizer Wirtschaft an wenig qualifiziertem Personal in den Bereichen Hauswirtschaft, Hotellerie und Gaststättengewerbe sowie Landwirtschaft.

Diese Zulassungspolitik trägt nicht den Ergebnissen neuerer Forschungsarbeiten Rechnung, wie jenen der OECD, die gewisse positive Auswirkungen der Migration auf die ärmsten Herkunftsländer nachweisen. Mehrere Studien zeigen insbesondere, dass die am wenigsten qualifizierten Migrantinnen und Migranten dazu neigen, mehr Geld in ihr Heimatland zu überweisen als die hoch qualifizierten Emigranten oder solche, die sich seit langem in einem Land des Nordens niedergelassen haben ${ }^{25}$.

\section{Eine immer restriktivere Asylpolitik}

Seit mehreren Jahrzehnten ist die politische Lage in der Schweiz durch die zunehmende Verschärfung der Politik zur Flüchtlingsaufnahme geprägt. Unter dem Druck der Volksabstimmungen und der politischen Agenda der nationalistischen Parteien ist die Asylpolitik der Schweiz zu einer der restriktivsten in Europa geworden ${ }^{26}$. Wir werden diesen Aspekt hier nicht behandeln, sondern verweisen interessierte Leserinnen und Leser auf den Beitrag von Gianni D’Amato im vorliegenden Dossier.

\section{Ein gespanntes politisches Klima}

Die für das Asylrecht eintretenden Nichtregierungsorganisationen haben stets rassistische Akte und Menschenrechtsverletzungen in der Schweiz (wie Gewalt gegen Ausländer oder unkontrollierte Aktionen bei der Rückführung abgewiesener Asylbewerber) angeprangert. Gewisse nationalistische Kreise und einige Medien zögern nicht, mit dem Finger auf die eine oder andere Ausländergemeinschaft $\mathrm{zu}$ zeigen und dadurch fremdenfeindliche und rassistische Tendenzen zu schüren. Die Wahrnehmung bezüglich einer Ausländergemeinschaft kann sich glücklicherweise mit der Zeit ändern: Die Sri-Lanker, deren Präsenz in der Schweiz Mitte der 80er Jahre grosses Misstrauen erregt hatte, wurden wegen ihrer Seriosität und ihres Arbeitseifers schliesslich geschätzt ${ }^{27}$. Jedoch ist das politische Klima in der Schweiz - wie auch in anderen europäischen Ländern der Öffnung nicht förderlich, und die politische Debatte wird oft eher emotional als sachlich geführt.

\section{Nachdruck auf Rückübernahmeabkommen}

Wie andere europäische Staaten hat die Schweiz Schwierigkeiten, die Rückschaffung abgewiesener Asylbewerber umzusetzen. Angesichts der Notwendig-

25 Siehe Kapitel 4 der OECD-Publikation La cohérence des politiques au service du développement: migrations et pays en développement, op. cit.

26 Für einen geschichtlichen Überblick über die Verschärfung der Schweizer Gesetze von Jahr zu Jahr verweisen wir auf die Kapitel über Migrationspolitik und Asylfragen im Jahrbuch Schweiz-Dritte Welt (Jahrgänge 1984-2002) und im Schweizerischen Jahrbuch für Entwicklungspolitik, Fakten und Statistiken (Jahrgänge 2003-2008).

27 Siehe beispielsweise die Analyse von Joëlle Moret, Denise Efionayi und Fabienne Stants, Diaspora sri lankaise en Suisse, Bern, Bundesamt für Migration, 2007, 156 S. 
keit, eine kohärente und effiziente Politik aller vom Migrations- und Rückkehrbereich betroffenen Departemente und Ämter zu erreichen, setzte der Bundesrat 2003 die Interdepartementale Arbeitsgruppe „Aussenpolitik im Migrations- und Rückkehrbereich" (IDAG) mit dem Bundesamt für Migration und der Politischen Abteilung IV des EDA ein. Über die Arbeiten dieser Gruppe wurde im darauffolgenden Jahr ein Bericht veröffentlicht ${ }^{28}$. Der Bericht bekräftigt das Engagement der Schweiz im internationalen Dialog und betont die Notwendigkeit, Migrationspartnerschaften und insbesondere Rückübernahmeabkommen mit den hauptsächlichen Herkunftsländern der Migranten abzuschliessen. Auf diese Weise wurden von der Schweiz rund vierzig bilaterale Rückübernahmeabkommen abgeschlossen ${ }^{29} .2003$ ging Bundesrätin Ruth Metzler noch weiter und versuchte, ein Transitabkommen mit dem Senegal auszuhandeln. Das Abkommen hätte es ermöglicht, dass afrikanische Asylbewerber zunächst in den Senegal zurückgeschickt würden, bis man ihre genaue Staatsangehörigkeit festgestellt hätte, um sie dann in ihr Herkunftsland zurückschicken zu können. Diesen Bemühungen war jedoch kein Erfolg beschieden, da sich das Parlament des Senegals infolge der Mobilisierung der Zivilgesellschaft weigerte, ein solches Abkommen zu ratifizieren.

\section{Hilfe für eine wirklich freiwillige Rückkehr?}

Das Instrument der Hilfe für eine freiwillige Rückkehr wird von der Schweiz als vorbildlich angesehen. Kann aber wirklich von einer Hilfe zur „freiwilligen“ Rückkehr die Rede sein? Die Betroffenen, das heisst die Mehrheit der abgewiesenen Asylbewerber, haben keine grosse Wahl: Sie können entweder in den Untergrund gehen, ihr Glück - mit geringen Erfolgsaussichten - in einem anderen europäischen Land versuchen, eine gewaltsame Rückschaffung in ihr Land riskieren, oder Hilfe annehmen und freiwillig in ihr Heimatland zurückkehren.

Das Instrument der Rückkehrhilfe hat sich vor allem in einem besonderen Kontext, jenem der Beendigung des Konflikts in den Staaten des früheren Jugoslawiens, als beispielhaft erwiesen. Von den 65000 Personen, die Rückkehrhilfe erhielten, waren 40000 aus dem Kosovo und 10000 aus Bosnien. Rückkehrhilfeprogramme dieser Art bestehen für rund zwanzig Länder, in manchen Fällen lediglich mit ein paar hundert „Kandidatinnen und Kandidaten“. Ist dieses Instrument aber wirklich für andere Situationen als für ein Gebiet bei Kriegsende geeignet? Wird die Wiedereingliederung anhaltend sein? Wie können die betroffenen Rückkehrerinnen und Rückkehrer hoffen, sich in einer Region dauerhaft wieder integrieren zu können, die beispielsweise eine hohe Arbeitslosenrate aufweist oder Opfer latenter interethnischer Konflikte ist?

Die Studien des Bundesamtes für Migration stellen die Synergien zwischen der vom BFM geleisteten individuellen Hilfe und den von der DEZA vor Ort durchgeführten Strukturhilfeprojekten heraus ${ }^{30}$. Solche Synergien zwischen Rück-

28 Interdepartementale Arbeitsgruppe „Aussenpolitik im Migrations- und Rückkehrbereich“, Schlussbericht der IDAG Migration, Bern, 2004, <http://www.eda.admin.ch/etc/medialib/downloads/edazen/ topics/migr.Par.0001.File.tmp/Schlussbericht_Interdept_AG_Migration_de.pdf $>$.

29 Die Liste dieser Abkommen ist im Internet erhältlich unter: <http://www.bfm.admin.ch/bfm/de/home/ themen/internationales/internationale_vertraege/ref_schweizerische.html>.

30 Betreffend die Evaluationsberichte verschiedener Rückkehrhilfeprogramme verweisen wir auf die Website des Bundesamtes für Migration: <http://www.bfm.admin.ch>. 
kehrhilfe und Entwicklungszusammenarbeit scheinen effektiv in jenen Ländern zu bestehen, in denen die DEZA mit einem breitgefächerten Instrumentarium und mit lokalen Kontakten bereits gut vertreten ist. Hingegen scheint es, dass sie in Ländern, in denen die DEZA wenig aktuelle Programme unterhält, schwieriger zu erreichen ist.

\section{Instrumentalisierung der Hilfe zur Eindämmung der Migration}

Es besteht die grosse Gefahr, die Entwicklungszusammenarbeit als Instrument einsetzen zu wollen, um die Partnerländer zu zwingen, ihre von der Schweiz abgewiesenen Staatsangehörigen wieder aufzunehmen. Der Versuch, die öffentliche Entwicklungshilfe von einer „guten Rückübernahmepraxis“ abhängig machen zu wollen, wurde mehrfach angeprangert.

In einem vor einiger Zeit veröffentlichten Interview erwiderte der frühere DEZA-Direktor Walter Fust auf die Frage, ob der Druck, die Schwerpunkte der Entwicklungszusammenarbeit in bestimmten Ländern oder Bereichen auch nach politischen Kriterien zu setzen, in den letzten Jahren zugenommen habe: „Eindeutig. Das schadet nicht, wenn es eine Debatte darüber gibt. Aber im Fokus muss die Reduktion der Armut stehen. Nimmt der Druck zu, die Entwicklungszusammenarbeit als Instrument zu anderen Zwecken zu benutzen, führt das sehr schnell zu einem Glaubwürdigkeitsverlust.“ Nach den angesprochenen „Zwecken“ befragt, fügte Walter Fust hinzu: „Zum Beispiel die Migrationspolitik. Wenn ein paar hundert Personen in ein Land zurückkehren müssen und wir wegen der Rückkehrhilfe daraus ein Schwerpunktland machen sollen, hat es mit Armutsreduktion nichts zu tun. Ebenso, wenn wir uns an irgendeiner Initiative beteiligen, nur um einer Grossmacht zu gefallen. Wir plädieren dafür, dass man nicht von Gelegenheit zu Gelegenheit hüpft, sondern die Qualität und die Kontinuität der Entwicklungszusammenarbeit durch Wirkung zum Tragen bringt. Man kann auf unseren Leistungen aussen- oder migrationspolitisch aufbauen, soll sie aber nicht zweckentfremden.“31

\section{Ansätze für eine aktivere internationale Zusammenarbeit}

Ein wichtiger Aspekt im Bericht der Weltkommission für Internationale Migration (GCIM) ist die Befürwortung von Politiken, die es erlauben, gegen die negativen Folgen der Migration anzugehen und gleichzeitig zu versuchen, die positiven Effekte der Migration in den Herkunftsländern der Migranten zu maximieren. Es geht nicht darum, die Entwicklungszusammenarbeit zur Eindämmung der Migrationsströme zu instrumentalisieren, sondern vielmehr darum, die Migration für die Entwicklung zu fördern.

Im Migrationsbereich erstellt die Entwicklungszusammenarbeit der Schweiz, wie die Entwicklungshilfeagenturen anderer OECD-Staaten, vor allem Projekte mit dem Ziel, den Zustrom von Migranten einzudämmen (Migrationsprävention, Rückkehrhilfe), den Menschenhandel zu bekämpfen und Krisensituationen durch humanitäre Einsätze nachzukommen. Bislang gibt es noch sehr wenig

31 „Entwicklungshilfe darf nicht instrumentalisiert werden“, Interview mit Walter Fust, Neue Zürcher Zeitung, 22. April 2008. 
Projekte, die auf einer positiveren Vision der Migration und auf neuen Formen der Unterstützung für Migrantengemeinschaften basieren. Nachstehend werden einige mögliche Ansätze für Projekte solcher Art aufgeführt.

\section{Unterstützung der Initiativen von Migrantenverbänden}

Eines der Grundprinzipien der Entwicklungszusammenarbeit ist, für die Bedürfnisse der Basis aufgeschlossen zu sein. Bei den Programmen der Schweiz im Migrationsbereich sind die Initiativen aber häufig „,von oben nach unten“ ergangen, wie zum Beispiel bei den vom Bundesamt für Migration initiierten Rückkehrhilfeprojekten. Um den Bedürfnissen der Basis Rechnung zu tragen, müsste man die Migrantengemeinschaften in der Schweiz besser kennen und ihren Bedarf mit ihnen zusammen festlegen; jedoch kennen sich die Migrantenverbände, die nichtstaatlichen Organisationen und die amtlichen Entwicklungshilfestellen nicht sehr gut. Wie steht es mit den prioritären Bedürfnissen der Migrantinnen und Migranten in der Schweiz? Wie können die Kosten für die Überweisungen der Migranten an ihre Familien gesenkt werden? Brauchen die Migranten Rückkehrhilfe? Erst aufgrund von Studien, die es ermöglichen, die wirklichen Bedürfnisse der Migranten besser zu ermitteln, können angepasste Projekte entwickelt werden. Statt einer direkten Beziehung zwischen dem Staat und den Migrantenverbänden könnten sich die NRO oder die kantonalen Föderationen für Entwickungszusammenarbeit ferner am besten imstande erweisen, Projekte in Zusammenarbeit mit den Migrantenverbänden auszuarbeiten. Dies schliesst jedoch nicht Formen der Unterstützung durch den Bund oder die Kantone für Initiativen der Diaspora aus. Auch hat das Weltforum für Migration und Entwicklung (GFMD) auf seiner Tagung in Brüssel die Errichtung dreiteiliger Partnerschaften zwischen Diaspora, Herkunftsländern und Aufnahmeländern der Migranten empfohlen.

\section{Ausgleich für Braindrain-fördernde Migrationspolitiken}

Gemäss der OECD berücksichtigen einige Politiken der OECD-Staaten ihre Auswirkungen auf die Entwicklungsländer nicht. Nach Zahlenangaben der Internationalen Organisation für Migration arbeitet einer von vier afrikanischen Ärzten in einem OECD-Staat, wohingegen es vielen afrikanischen Ländern an menschlichen Ressourcen in ihren Gesundheitssystemen mangelt. Die OECDStaaten haben diese Abwanderung qualifizierter Arbeitskräfte oftmals gefördert durch die Erteilung von Arbeitsbewilligungen für Fachkräfte des Gesundheitssektors, die in den Krankenhäusern des Nordens fehlen. Auch wenn es schwierig ist, die Tendenz umzukehren und zu verhindern, dass ein ägyptischer Arzt es vorzieht, in einem Bereitschaftsdienst in Genf statt in Kairo zu arbeiten, könnte der Verlust für das Herkunfstland jedoch durch andere Mittel ausgeglichen werden, wie eine gezielte Hilfe für den Gesundheitssektor des betreffenden Landes, die Stärkung seiner Ausbildungskapazitäten oder die Bereitstellung von Kompetenzen, indem dem man es dem Arzt gestattet, zeitweilig in seinem Herkunftsland zu arbeiten. 


\section{Neue Formen temporärer oder zirkulärer Migration finden}

Es ist wichtig, Arbeitnehmerinnen und Arbeitnehmern, die eine Berufstätigkeit in der Schweiz ausüben wollen, die Möglichkeit eines temporären Aufenthalts im Land bieten zu können, wie auch Studierenden, die eine Berufserfahrung in der Schweiz erlangen möchten, bevor sie in ihr Heimatland zurückkehren. Das Weltforum für Migration und Entwicklung hat in Brüssel darauf hingewiesen, dass gemeinsame Abkommen zwischen den Herkunfts- und Zielländern der Migranten dazu beitragen würden, den Schutz der Rechte der temporären Arbeitnehmer, namentlich der am wenigsten qualifizierten Migranten, zu stärken und dadurch deren Beiträge an ihr Herkunftsland zu verbessern. Die Zielländer könnten zum Beispiel flexiblere Massnahmen für die Zulassung in der Schweiz und die Erlangung einer Arbeitsbewilligung einführen, und die Herkunftsländer würden sich zum Ausgleich verpflichten, die Migranten anzuhalten, ihre Kompetenzen in ihrem eigenen Land einzusetzen. Jedoch könnte sich die Debatte über neue mögliche Formen zeitweiliger Zulassung von Migranten in der Schweiz - in Anbetracht des früheren, stark umstrittenen Saisonnierstatuts - als schwierig erweisen.

\section{Einsatz der Kompetenzen von Migranten für Projekte im Herkunftsland}

Mehrere bestehende Programme der Entwicklungszusammenarbeit haben zum Ziel, die Bereitstellung von Kompetenzen ausländischer Gebietsansässiger in den Ländern des Nordens zu fördern, um Projekte im Süden zu unterstützen. So könnte etwa ein Arzt in seinem Herkunftsland vorübergehend lehren und arbeiten. Die IOM-Projekte „Migration für die Entwicklung Afrikas“ („Migration pour le développement de l'Afrique“, MIDA) mobilisieren beispielsweise die afrikanische Diaspora in Europa durch zeitweilige Einsätze im Gebiet der Grossen Seen, in Ghana und im Senegal ${ }^{32}$. Desgleichen fördert das Programm TOKTEN („Transfer of Knowledge Through Expatriate Nationals“) des Entwicklungsprogramms der Vereinten Nationen (UNDP) die Übermittlung von Wissen durch Staatsangehörige im Ausland.

Auch wenn Evaluationen noch nachzuweisen haben, dass solche Initiativen eine merkliche Auswirkung auf die Entwicklung des Partnerlandes im Süden - zum Beispiel durch die Verbesserung der sanitären Lage - haben können, und auch wenn diese Programme nicht die internationale Zusammenarbeit ersetzen können, wäre es interessant, ihre Machbarkeit in der Schweiz zu prüfen.

\section{Unterstützung der Überweisungen von Migranten zur Förderung der Entwicklung}

Viele Studien haben in den letzten Jahren die Bedeutung der Geldüberweisungen der Migranten hervorgehoben und die positive Wirkung eines Teils dieser

32 Tamara Keating, „Migration et développement pour l'Afrique : mobiliser les ressources de la diaspora pour le renforcement des capacités dans les pays d'origine“, in Schweizerisches Rotes Kreuz (Hrsg.), Migration, ein Beitrag zur Entwicklung ?, Zürich, Seismo, 2007. Siehe auch die Website des MIDAProgramms für die Grossen Seen, <http://www.belgium.iom.int/Mida2/presentation.asp>. 
Überweisungen auf die Entwicklung und die Armutsbekämpfung nachgewie$\operatorname{sen}^{33}$. Im Anschluss an eine in Auftrag gegebene Studie über die Geldüberweisungen der Migranten nach Serbien evaluiert das SECO die Möglichkeiten einer Zusammenarbeit in diesem Bereich ${ }^{34}$. Norwegen hat ein Pilotprojekt eingeführt, um die Geldüberweisungen von Migranten nach Pakistan zu unterstützen: Ab einer Zahlung von 20000 Franken durch ein Netzwerk pakistanischer Migranten verdoppelt der Staat den Betrag, indem er zusätzliche 20000 Franken hinzuzahlt $^{35}$. Dieses System erinnert an das mexikanische System „Tres por uno“, das es Migrantenverbänden in den Vereinigten Staaten ermöglicht, bei einer Geldüberweisung den Betrag zu verdreifachen, indem zum Beitrag der Migranten noch ein gleichwertiger Betrag seitens des mexikanischen Lokalstaats und des Zentralstaats hinzukommt. Warum sollte man nicht einen zusätzlichen Beitrag dieser Art durch den Schweizer Staat ins Auge fassen? Oder warum nicht Mittel finden, um die Transaktionskosten bei den Geldüberweisungen der Migranten zu senken? Die Entwicklungszusammenarbeit sollte zudem Migrantengemeinschaften, die dies wünschen, bei der Errichtung konkreter Projekte in ihren Herkunftsgebieten helfen, um das Unternehmertum, die Gründung kleiner Unternehmen oder den Zugang zu Krediten für einzelne lokale Projekte zu unterstützen. Es geht für den Staat nicht darum, sich die Verwaltung privater Finanzflüsse anzueignen, sondern die Initiativen von Migranten zugunsten ihres Herkunftsgebiets zu unterstützen.

\section{Migrationspartnerschaften als Allheilmittel?}

Die Errichtung von Migrationspartnerschaften soll den Dialog zwischen der Schweiz und dem Herkunfts- oder Transitland der Migranten verbessern ${ }^{36}$. Das neue Bundesgesetz über die Ausländerinnen und Ausländer (AuG) vom 16. Dezember 2005 legt im Artikel 100 fest: „Der Bundesrat fördert bilaterale und multilaterale Migrationspartnerschaften mit anderen Staaten. Er kann Abkommen abschliessen, um die Zusammenarbeit im Migrationsbereich zu stärken sowie die illegale Migration und deren negative Folgen zu mindern.“

Es bleibt zu wünschen, dass die geplanten Massnahmen es erlauben werden, den Interessen der Herkunftsländer der Migranten besser Rechnung zu tragen, und dass sie nicht wieder auf die Mittel zur Eindämmung der Migration ausgerichtet werden. In der Tat ist von einer polizeilichen und institutionellen Verstärkung im Partnerland die Rede, um diesem zu helfen, die Migrationsströme besser zu bewältigen, auch davon, den Maghrebstaaten zu helfen, die Transitmigration auf ihrem Gebiet besser in den Griff zu bekommen. Die vom Bundesrat gegenüber dem Westbalkan (Kosovo, Bosnien-Herzegowina und Serbien) eingeführte Strategie erwähnt beispielsweise Massnahmen zur Verstärkung der lokalen und nationalen Behörden bei der Migrationsbewältigung, dem Kampf gegen den Migrantenschmuggel und der Rückkehrhilfe. Doch wie werden die Repräsentanten der

\footnotetext{
33 Siehe die Beiträge von Claude Auroi und von Alessandro Monsutti (Hawala-System) in diesem Dossier.

34 Siehe den Artikel von Mathias Lerch zu den Überweisungen der in der Schweiz niedergelassenen serbischen Migranten in diesem Dossier.

35 Der Bund, 22. Juli 2008.

36 Siehe den Beitrag von Therese Liechti und Monica Budowski in diesem Dossier.
} 
Schweiz reagieren, wenn die Vertreter der Partnerländer temporäre Aufenthaltsmöglichkeiten für ihre Staatsangehörigen zu Berufszwecken in der Schweiz fordern? Wie werden sie reagieren, wenn die Herkunftsländer eine bessere Bekämpfung der Ausbeutung der Arbeitsmigranten in der Schweiz verlangen? In diesem Kontext ist es wichtig, einen besserer Dialog zwischen den Herkunftsländern der Migranten und der Schweiz einzuleiten.

\section{Schlussbemerkungen}

Die Entwicklungszusammenarbeit wurde allzu oft als ein Mittel unter anderen angesehen, um den Zustrom von Migranten in die Schweiz soweit wie möglich einzudämmen und abgewiesenene Asylbewerber dazu zu bewegen, ,freiwillig“ in ihr Land zurückzukehren. Jedoch erweist es sich als illusorisch, die Migrationsströme verhindern zu wollen. Es ist an der Zeit, Strategien der Entwicklungszusammenarbeit und Migrationspolitiken zu konzipieren, die der Komplexität der Migrationsphänomene und ihren positiven Aspekten besser Rechnung tragen.

In der Schweiz muss der Dialog zwischen der Bundesverwaltung, den Kantonalbehörden und der betroffenen Zivilgesellschaft (Migrantenverbände, Menschenrechtsorganisationen, NRO für Entwicklungszusammenarbeit und humanitären Hilfe) verbessert werden. Auch muss die Teilnahme der Migrantenverbände am nationalen und internationalen Dialog nicht nur im Hinblick auf die Integration der Migranten, sondern auch auf die Beziehungen mit ihrem Herkunftsgebiet oder Heimatland gestärkt werden.

In den Ländern des Nordens herrscht eine überwiegend negative Sicht des Migrationsphänomens vor. Die Entwicklungszusammenarbeit kann durch konkrete Projekte zur Unterstützung der Migranten, durch Anwaltschaft, durch eine bessere Berücksichtigung der Komplexität der Migrationsströme und durch die Bekämpfung ihrer unerwünschten Auswirkungen einen weitaus konstruktiveren Ansatz der Migrationsproblematik fördern.

\section{Bibliographie}

Bundesamt für Migration, Migrationsbericht 2007, Bern, BFM, April 2008.

Dayton-Johnson, Jeff und Louka T. Katseli, Migration, aide et commerce: plus de cohérence en faveur $d u$ développement, Cahier de politique économique, Nr. 28, Paris, OCDE, 2006.

DEZA, Internationale Zusammenarbeit und Migration, Entwicklungspolitische Schriften der DEZA, Nr. 4, Bern, DEZA, April 2001.

DEZA, „Migration: Auch Chancen, nicht nur Herausforderungen“, Dossier, Eine Welt, Nr. 1, März 2008.

DEZA, „Migration und Entwicklungszusammenarbeit“, Dossier, Eine Welt, Nr. 2, Mai 1999.

Longchamp, Claude, Monica Aebersold, Bianca Rousselot und Silvia Ratelband-Pally, Sans Papiers in der Schweiz: Arbeitsmarkt, nicht Asylpolitik ist entscheidend. Schlussbericht im Auftrag des Bundesamtes für Migration, Bern, GFS, Februar 2005.

Moret, Joëlle, Denise Efionayi und Fabienne Stants, Diaspora sri lankaise en Suisse, Bern, Bundesamt für Migration, 2007, $156 \mathrm{~S}$.

OCDE, La cohérence des politiques au service du développement. Migrations et pays en développement, Paris, OCDE, 2007, $164 \mathrm{~S}$.

Organisation internationale pour les Migrations, Partenariats migratoires : présentation du concept dans les contextes international et suisse, Bern, OIM, 2007, $81 \mathrm{~S}$. 
Schweizerisches Rotes Kreuz (Hrsg.), Migration, ein Beitrag zur Entwicklung?, Zürich, Seismo, 2007, $274 \mathrm{~S}$.

\section{Internetadressen}

Eidgenössisches Departement für auswärtige Angelegenheiten (EDA), Direktion für Entwicklung und Zusammenarbeit (DEZA), Migration und Projekte: <http://www.deza.admin.ch/de/Home/Themen/ Migration>.

Eidgenössisches Departement für auswärtige Angelegenheiten (EDA), Politische Abteilung IV, Massnahmen im Migrationsbereich : <http://www.eda.admin.ch/eda/de/home/topics/migr.html>.

Eidgenössisches Justiz- und Polizeidepartement (EJPD), Bundesamt für Migration (BFM), Rückkehrförderung: <http://www.bfm.admin.ch/bfm/de/home/themen/rueckkehr/rueckkehrfoerderung.html>.

Global Commission on International Migration: <http://www.gcim.org $>$. 